\title{
Quantitative Phenotype Scan Statistic (QPSS) reveals rare variant associations with Alzheimer's disease endophenotypes
}

\author{
CURRENT STATUS: UNDER REVIEW \\ BMC Medical Genetics D BMC Series \\ Yuriko Katsumata \\ University of Kentucky \\ katsumata.yuriko@uky.eduCorresponding Author \\ ORCiD: https://orcid.org/0000-0002-0188-8094 \\ David W. Fardo \\ University of Kentucky
}

DOI:

10.21203/rs.2.20987/v1

SUBJECT AREAS

Medical Genetics

KEYWORDS

QPSS, WGS, ADNI, Localization 
Abstract

Background: Current sequencing technologies have provided for a more comprehensive genome-wide assessment and have increased genotyping accuracy of rare variants. Scan statistic approaches have previously been adapted to genetic sequencing data. Unlike currently-employed association tests, scan-statistic-based approaches can both localize clusters of disease-related variants and, subsequently, examine the phenotype association within the resulting cluster. In this study, we present a novel Quantitative Phenotype Scan Statistic (QPSS) that extends an approach for dichotomous phenotypes to continuous outcomes in order to identify genomic regions where rare quantitative-phenotype-associated variants cluster.

Results: We demonstrate the performance and practicality of QPSS with extensive simulations and an application to a whole-genome sequencing (WGS) study of cerebrospinal fluid (CSF) biomarkers from the Alzheimer's Disease Neuroimaging Initiative (ADNI). Using QPSS, we identify regions of rare variant enrichment associated with levels of AD-related proteins, CSF A 1 1-42 and p-tau 181P. Conclusions: QPSS is implemented under the assumption that causal variants within a window have the same direction of effect. Typical self-contained tests employ a null hypothesis of no association between the target variant set and the phenotype. Therefore, an advantage of the proposed competitive test is that it is possible to refine a known region of interest to localize disease-associated clusters. The definition of clusters can be easily adapted based on variant function or annotation.

\section{Full-text}

Due to technical limitations, full-text HTML conversion of this manuscript could not be completed. However, the manuscript can be downloaded and accessed as a PDF.

\section{Supplementary Files}

This is a list of supplementary files associated with this preprint. Click to download. SupplementaryMaterials.pdf 